\title{
PERFIL DA FARMÁCIA CLÍNICA EM PACIENTES RENAIS EM TRATAMENTO DE HEMODIÁLISE EM UMA CLÍNICA PARTICULAR EM BELÉM DO PARÁ
}

\author{
Bárbara Roberta da Costa Loiola ${ }^{1}$, Suzana Moraes Viégas ${ }^{2}$ e Eliene dos Santos da Silva Costa ${ }^{3}$ \\ ${ }^{1}$ Escola Superior da Amazônia, ${ }^{2}$ Faculdade Venda Nova do Imigrante, ${ }^{3}$ Centro Universitário Fibra \\ E-mail para correspondência: elienessilva@yahoo.com.br
}

Submetido em: 11/06/2021 e aprovado em: 02/08/2021

\begin{abstract}
RESUMO
Introdução: A doença renal crônica está cada vez mais prevalente e os pacientes em tratamento de hemodiálise formam um grupo específico de observações em farmácia clínica devido a presença de patologias associadas, o que leva ao uso de polifarmácia e aumenta a possibilidade de haver mais interações medicamentosas e agravos à saúde dos pacientes. Objetivos: $\mathrm{O}$ objetivo desse trabalho foi montar um perfil da farmácia clínica como área de atuação do farmacêutico na equipe multidisciplinar e identificar e classificar possíveis interações medicamentosas (IM), através da análise dos prontuários dos pacientes em tratamento de hemodiálise em uma clínica particular na cidade de Belém do Pará. Foram analisados 50 prontuários de pacientes e após a aplicação do método de análise de conteúdo (Bardim), foi produzido uma proposta de memento terapêutico para a clínica de pacientes renais baseado nos medicamentos prescritos encontrados. Resultados e discussão: observou-se em 32 prontuários, 25 classes de medicações diferentes prescritas. O sexo feminino $(56,25 \%)$ prevaleceu, comparado ao sexo masculino (43,75\%). Resultado demonstrou $12 \%$ de interações/associações, benéficas e maléficas. Conclusão: Com análise das interações medicamentosas encontradas, concluiu-se que a presença do farmacêutico é indispensável na equipe multidisciplinar, para acompanhamento dessas possíveis interações com objetivo de minimizar as reações indesejáveis, trazendo benefícios ao tratamento do paciente renal crônico.

Palavras-chave: Medicamento, interação, prontuários, renal, farmacêutico.
\end{abstract}

\begin{abstract}
Introduction: Chronic kidney disease is becoming more prevalent and patients undergoing hemodialysis form a specific group of observations in clinical pharmacy due to the presence of other associated pathologies, which leads to the use of polypharmacy and increases the possibility of more drug interactions and health problems of patients. Aims: The objective of this work was to build a profile of the clinical pharmacy as an area of action of the pharmacist in the multidisciplinary team and to identify and classify possible drug interactions (MI) through the analysis of medical records of patients undergoing hemodialysis treatment in a private clinic in the city of Belém from Pará. Methods: Fifty patient files were analyzed and after the application of the content analysis method (Bardim), a proposal was made of memento therapeutics for the clinic of renal patients based on the prescribed drugs found. Results and discussion: It were observed in 32 medical records, 25 different classes of prescribed medications. The female gender $(56.25 \%)$ prevailed, compared to the male gender $(43.75 \%)$. Results showed $12 \%$ of interactions/associations, both beneficial and harmful. Conclusions: With the analysis of the interactions found, from the results, the necessary presence of the pharmacist in the multidisciplinary team was concluded, to follow up on possible interactions that could be minimized, bringing more benefits than harm to the treatment of the chronic renal patient.
\end{abstract}

Keywords: Drug, Interaction, Medical records, Renal, Pharmaceutical. 


\section{INTRODUÇÃO}

A polifarmácia (uso de vários medicamentos) é uma prática cada vez mais comum na sociedade moderna, devido ao aumento progressivo da expectativa de vida e pela alta prevalência de doenças crônicas não transmissíveis $(\mathrm{DCNT})^{(1)}$.

Na prática clínica, a polifarmácia é vista com frequência e podem estar relacionadas a interações medicamentosas (IM), entre outros fatores ${ }^{(2)}$. As interações medicamentosas, são alterações que ocorrem nos efeitos dos fármacos, ou seja, quando dois ou mais medicamentos são administrados concomitantemente a um paciente, agindo de forma independente ou entre si, levando ao aumento ou diminuição do efeito terapêutico ou tóxico de um ou de outro medicamento $^{(3)}$.

De acordo com estudos, pacientes com várias patologias, como disfunção renal, que geralmente, fazem uso de muitos medicamentos são os mais suscetíveis. A doença renal crônica (DRC) é definida como alterações na taxa de filtração glomerular e/ou presença de lesões parenquimatosas mantidas por no mínimo três meses ${ }^{(4)}$. Com o diagnóstico de DRC confirmado, o paciente dá início ao tratamento conservador (medicamentos, dieta e estilo de vida), caso o quadro não melhore, a evolução da doença pode leva-lo a insuficiência renal crônica (IRC) ${ }^{(5)}$.

A IRC é forma mais grave da doença, no qual o tratamento de terapia renal substitutiva (TRS) ou também chamado de hemodiálise, é a única alternativa capaz de manter funções vitais dos pacientes até conseguir algum doador compatível para o transplante renal ${ }^{(5)}$.

De acordo com o ministério da saúde as taxas regionais de pessoas que se encaixam em alguma TRS e que apresenta maior índice é a região Sudeste, com 236 pessoas a cada 100 mil, seguida pela região Centro-oeste (229 por 100 mil da população) e região Sul (208 por 100 mil da população) ${ }^{(6)}$.

Adicionalmente, segundo a Sociedade Brasileira de Nefrologia (SBN) as doenças primárias frequentemente diagnosticadas que levam à DRC são: diabetes mellitus (30\%), hipertensão arterial (34\%), seguidos pela glomerulonefrite crônica (9\%) e rins policísticos (4\%). Além disso, o tratamento para pacientes diagnosticados com a IRC pode ser classificado de duas maneiras: a Hemodiálise e a Diálise peritoneal ${ }^{(7)}$.

Em relação aos serviços de diálise, a Resolução $n^{\circ} 500$, dispõe sobre as atribuições do farmacêutico no âmbito desta atividade, de natureza pública ou privada, autorizando-o e 
descrevendo as funções que o farmacêutico pode realizar dentro de uma clínica/hospital que ofereçam os serviços de diálise ${ }^{(8)}$.

Com base na resolução descrita acima, uma das atribuições do profissional farmacêutico na diálise, é a orientação aos pacientes a aderirem ao tratamento, que podem ser longos e dolorosos $^{(9)}$. Com isso, o farmacêutico pode possibilitar a melhora nos efeitos terapêuticos e ainda diminuir a probabilidade de aparecimento de reações adversas e as possíveis interações medicamentosas $^{(10)}$.

Nesse sentido, ao ser levado em conta a criticidade dos pacientes atendidos na clínica de hemodiálise, assim como a particularidade de seu tratamento, faz-se necessário caracterizar as IM com finalidade de diminuir os riscos relacionados ao uso de medicamentos, promovendo a segurança do paciente.

O presente artigo apresenta como objetivo principal detectar possíveis interações medicamentosas presentes nas prescrições médicas que constam nos prontuários dos pacientes renais atendidos em uma clínica particular de Belém.

\section{MÉTODOS}

A pesquisa teve um caráter transversal retrospectivo qualitativa do tipo descritivo ${ }^{(2)}$. O estudo foi desenvolvido em uma clínica particular, voltada ao tratamento de pacientes renais crônicos, na cidade de Belém, na qual foram selecionados 50 prontuários de pacientes, no período de Janeiro de 2017 a Julho de 2018.

Foram incluídos na pesquisa, prontuários de pacientes do sexo masculino e feminino, na faixa etária de 18 a 65 anos, ainda em tratamento na clínica. Por outro lado, foram excluídos os prontuários com dificuldade de leitura (ilegíveis), prontuários de pacientes que vieram a óbito, prontuários de pacientes transplantados ou transferidos, prontuários de pacientes com doenças virais (HIV e HEPATITE) e prontuários de pacientes possivelmente grávidas.

Os dados foram coletados através do prontuário institucional e analisados utilizando à análise de conteúdo, que é uma técnica que determina as características metodológicas (objetividade, sistematização e inferência). A técnica utilizada é composta por três etapas: préanalítica (organização e exploração do material analisado); exploração do material (codificação do material); e o tratamento dos resultados. 


\section{RESULTADOS E DISCUSSÃO}

De acordo com os resultados, dos 50 prontuários analisados, apenas 32 foram utilizados para a elaboração deste trabalho, isso porque, 18 prontuários apresentavam dados incompletos (sem escolaridade, sem medicação prescrita, entre outras informações).

Após análise criteriosa, foram encontrados um total de 25 classes medicamentos diferentes prescritos. Entre as classes mais prevalentes destacam-se os anti-hipertensivos, antianêmicos e suplemento vitamínico (Tabela 1). O perfil epidemiológico foi apresentado na tabela 2, e as patologias mais frequentes, são apresentadas na tabela 3.

Tabela 1: Medicamentos mais prescritos nos prontuários.

\begin{tabular}{ccc}
\hline Medicamentos & Indivíduos (n) & $\%$ \\
\hline Anti-hipertensivos & 20 & $67 \%$ \\
Nifedipino e Anlodipino & & \\
Antianêmicos & 6 & $20 \%$ \\
Eritropoitina e Noripurum ${ }^{\circledR}$ & 4 & $13 \%$ \\
Suplemento vitamínico & 4 & \\
Complexo B & & \\
\hline
\end{tabular}

Fonte: Os autores

Tabela 2: Perfil epidemiológico dos pacientes.

\begin{tabular}{|c|c|c|}
\hline Variável & Indivíduos (n) & $\%$ \\
\hline \multicolumn{3}{|l|}{ Gênero } \\
\hline Masculino & 14 & 43,7 \\
\hline Feminino & 18 & 56,3 \\
\hline \multicolumn{3}{|l|}{ Faixa etária } \\
\hline $18-40$ anos & 9 & 28,1 \\
\hline $40-65$ anos & 23 & 71,9 \\
\hline \multicolumn{3}{|l|}{ Área em que reside } \\
\hline Capital do estado & 23 & 71,9 \\
\hline Interior do estado & 9 & 28,1 \\
\hline \multicolumn{3}{|l|}{ Escolaridade } \\
\hline Analfabeto & 1 & 3,1 \\
\hline Nível fundamental & 24 & 75 \\
\hline Nível superior & 7 & 21,9 \\
\hline
\end{tabular}

Fonte: Os autores 
Tabela 3: Patologias mais frequentes.

\begin{tabular}{ccc}
\hline Patologia & Indivíduos (n) & $\%$ \\
\hline Hipertensão & 17 & 53,1 \\
Masculino & 9 & \\
Feminino & 8 & 18,8 \\
Diabetes + Hipertensão & 6 & 28,1 \\
Masculino & 1 & \\
Feminino & 5 & \\
Outras patologias & 9 & \\
Masculino & 4 & 5
\end{tabular}

Fonte: Os autores

Estudos demonstraram que pacientes portadores de diabetes e hipertensão, podem evoluir para insuficiência renal crônica ${ }^{(5,11)}$. Um estudo feito em São Paulo demonstrou que de 37 pacientes dialíticos, 20 eram diabéticos e hipertensos, 7 eram apenas hipertensos, 1 apenas diabético e 9 não possuíam nenhuma das doenças ${ }^{(11)}$. Em nosso estudo, observamos que de 32 pacientes dialíticos, 17 eram hipertensos, 6 eram diabéticos e hipertensos e 9 apresentaram outras doenças além do diabetes e da hipertensão.

Em 2015, um estudo realizado em Taubaté-SP, evidenciou que pessoas do sexo feminino (55\%), eram prevalentes dentre os pacientes dialíticos ${ }^{(12)}$. Nas pesquisas de HIGA et al (2008), DANIEL et al (2019) e BIANCA et al (2007), obteve prevalecia do sexo masculino em 75\%, $54 \%, 75 \%$, respectivamente ${ }^{(5,13,14)}$. A pesquisa atual corrobora com o primeiro estudo citado, pois também foi observado que o sexo feminino $(56,25 \%)$ prevaleceu, comparado ao sexo masculino $(43,75 \%)$. As mulheres têm mais infecções urinárias durante a idade fértil que os homes. Por outro lado, outros estudos apontam a prevalência do sexo masculino dentre os pacientes dialíticos. Pode-se presumir que o resultado apresentado, possa ser em decorrência da busca maior de mulheres aos serviços de saúde, proporcionando diagnósticos precoces.

A média de idade entre os pacientes em tratamento dialítico encontrado foi de 47,96 anos, coincidindo com o estudo feito em Itabuna-BA, onde a média de idade dos pacientes foi de 49,8 $\operatorname{anos}^{(15)}$. Podendo-se observar assim que a maioria dos pacientes portadores da doença renal crônica são os de idade ainda produtiva para o país ${ }^{(15,16)}$. 
O presente estudo apresentou maior frequência de hipertensão em homens, resultado semelhante ao observado em Goianésia-GO, onde os autores relataram que entre pacientes dialíticos, $65 \%$ eram do sexo masculino ${ }^{(17,18)}$.

Quanto as potenciais interações medicamentosas observadas (quadro 1), observou-se uma interação benéfica na associação de Anlodipino e Nifedipino, na qual o resultado é uma hipotensão arterial, pois os dois são da mesma classe farmacológica (bloqueadores de canais de cálcio) podendo assim, a dose ficar na faixa terapêutica e o efeito ser benéfico ao paciente. Porém em um estudo feito no Rio Grande do Sul, a administração concomitante dessas medicações, mostrou que o efeito foi potencializado com isso resultando em efeitos indesejados $^{(19,20)}$.

Quadro 1: Potenciais interações medicamentosas encontrados nos prontuários.

\begin{tabular}{|c|c|}
\hline Potencial interação medicamentosa & Gravidade \\
\hline Nifedipino e Anlodipino & Benéfica \\
\hline Nifedipino e Ácido Acetil Salićlico & Moderada \\
\hline Amitriptilina e Topiramato & Moderada \\
\hline
\end{tabular}

A interação de Nifedipino e Ácido Acetil Salicílico (AAS), pode elevar a pressão arterial, pois o AAS age como um antagonista, inibindo a enzima COX. Esse bloqueio pode fazer com que o medicamento anti-hipertensivo (Nifedipino) tenha parcial ou nenhum efeito sobre a pressão $\operatorname{arterial}^{(21)}$.

Já a interação da Amitriptilina com o Topiramato, pode produzir um aumento da concentração plasmática da Amitriptilina ${ }^{(22)}$.

As medicações prescritas ao paciente renal crônico necessitam de um acompanhamento rigoroso do profissional farmacêutico, objetivando identificar e intervir em possíveis problemas relacionados aos medicamentos, como as interações medicamentosas ${ }^{(23)}$.

\section{CONCLUSÃO}

Nesse estudo foi possível identificar um baixo número de interações medicamentosas, todavia, mais estudos devem ser efetuados com pacientes dialíticos, para identificação das interações medicamentosas mais frequentes e o risco destas para o paciente. 


\section{REFERÊNCIAS BIBLIOGÁFICAS}

1. Silveira EA, Dalastra L, Pagotto V. Polifarmácia, doenças crônicas e marcadores nutricionais em idosos. Rev Bras Epidemiol. 2014; 7(4): 818-829.

2. Neto M, Costa G, Nascimento P. Análise de Prescrições dos Pacientes Submetidos à Hemodiálise e Avaliação de Possíveis Interações Medicamentosas. JIC-Jornada de Pesquisa e Iniciação Científica. 2012; 3(3).

3. Marquito AB, Fernandes NMS, Colugnati FAB, Paula RB. Interações Medicamentosas Potenciais em Pacientes com Doença Renal Crônica. Jornal Brasileiro de Nefrologia. 2013; 36 (1): 26-34.

4. Bastos MG, Kirsztajn GM. Doença Renal Crônica: importância do diagnóstico precoce, encaminhamento imediato e abordagem interdisciplinar estruturada para melhora do desfecho em pacientes ainda não submetidos à diálise. J bras nefrol. 2011. 93-108.

5. Higa K, Kost MT, Soares DM, Morais MC, Polins BRG. Qualidade de vida de pacientes portadores de insuficiência renal crônica em tratamento de hemodiálise. Acta Paulista de Enfermagem. 2008; 21(número especial): 203-206.

6. Hoefler R, Wannmacher L. Interações de medicamentos. Ministério da Saúde. 2010. 1-14.

7. Sociedade Brasileira de Nefrologia [internet]. Tratamento conservador [acesso em $21 \mathrm{abr}$ 2018]. Disponível em: https: www.sbn.org.br.

8. Conselho Federal de Farmácia [internet]. Resolução da Diretoria Colegiada- RDC N 500, de 19 de Jeneiro de 2009. Dispõe sobre as Atribuições dos Farmacêuticos no Âmbito dos Serviços de Diálise, de natureza pública ou privada. 2009 [acesso em 19 abr 2018]. Disonível em: https: www.cff.org.br.

9. Terra FS, Costa AMDD, Figueiredo ET, Morais AM, Costa MD, Costa RD. Adesão ao tratamento farmacológico de uso diário de pacientes renais crônicos submetidos à hemodiálise. Rev Bras Clin Med. 2010; 8 (2): 119-24.

10. Lavrins CFS. A importância do profissional farmacêutico na melhoria da qualidade de vida dos pacientes diabéticos. Rio Verde, GO 2016.

11. Castro Júnior, Soares, Barbosa et al. Prevalência de Hipertensão Arterial e Diabetes Melitus em pacientes com doença renal crônica em ambulatório de cardiologia. Revista Cerus. 2017; 9 (3): 2-20.

12. Silva, Resende, Resende et al. Perfil clínico e sociodemográfico dos pacientes hemodialíticos de uma unidade de hemodiálise de Taubaté-SP. Educação Ciência Para a Cidadania Global. 2016. 1-5.

13. Teixeira DA, Hott RC, Moreira MCH, Moreira LE. A importância clínica na avaliação da adesão terapêutica de pacientes insuficientes renais crônicos atendidos em um hospital no nordeste de minas gerais. Revista Saúde dos Vales. 2019; 1(1).

14. Polins BRG, Higa K, Kost MT, Soares DM, Morais MC. Qualidade de vida de pacientes portadores de insuficiência renal crônica em tratamento de hemodiálise. Acta Paul Enferm. 2007; 21: 3-6. 
15. Oliveira CS, Silva EC, Ferreira LW, Skalinski LM. Perfil dos pacientes renais crônicos em tratamento hemodialítico. Revista Baiana de Enfermagem. 2015; 29 (1).

16. Soares, Aguiar, Furtado et al. Prevalência de hipertensão arterial e diabetes mellitus em portadores de doença renal crônica em tratamento conservador do Serviço Ubaense de Nefrologia. Revista Científica FAGOC-Saúde. 2018; 2(2): 21-26.

17. Fonseca IC, Rodrigues AMR, Machado KMSH. Insuficiência renal Crônica na Cidade de Goianésia-GO. Congresso Interdisciplinar. 2017. ISSN: 2595-7732.

18. Maia JC, Silva GF, Silva OM. Interações Medicamentosas em Pacientes de Hemodiálise: Conhecimento dos pacientes, prática da automedicação e o papel da enfermagem na orientação. Universidade do Estado de Santa Catarina. Seminário de Iniciação Científica. SC. 2017.

19. Agência Nacional de Vigilância Sanitária homepage na internet]. Bulário [acesso em 09 abr 2018]. Disponível em http: www. portal.anvisa.gov.br.

20. Bandeira, Rodigues, Rosa et al. Atenção Domiciliar Multiprofissional no Cuidado ao Idoso: Um Enfoque para o Uso Correto de Medicamentos - Relato de Caso. Ijuí, 2014.

21. Nascimento DM, Pigoso AA. Interação Medicamentosa entre Anti-hipertensivo e Antiinflamatório não esteroidais. Revista Científica da FH/UNIARARA. São Paulo. 2013; 1: 1417.

22. Hoefler R, Wannmacher L. Interações de medicamentos. Ministério da Saúde.2010. 1-14.

23. Seibt KS. A Importância da Atuação Farmacêutica em Pacientes com Insuficiência Renal Crônica em Tratamento Hemodialítico. Ariquemes: Faculdade de Educação e Meio Ambiente, 2013. Trabalho de conclusão de curso de Bacharelado em Farmácia. 